\title{
A llama-derived antibody for flu
}

Every year, seasonal influenza epidemics lead to 3-5 million cases of severe illness and approximately 300,000-650,000 deaths globally. Manufacturing an effective vaccine requires correctly predicting which influenza strains will dominate in the next flu season, and even well-formulated vaccines are less efficacious in the elderly, young children and immunocompromised individuals. Prophylaxis using broadly neutralizing antibodies, which bind conserved epitopes in multiple influenza viruses, could augment traditional vaccination by protecting vulnerable populations. Writing in Science, Laursen et al. ${ }^{1}$ describe a broadly neutralizing antibody format that combines four llama single-domain antibodies. In vitro, the fusion potently neutralizes 60 influenza A and $B$ viruses. Delivered to mice by intranasal adeno-associated virus (AAV), it confers robust protection against lethal doses of several influenza virus strains.

Llama and other camelid immunoglobulin $\mathrm{G}$ antibodies consist of only heavy chains containing a single variable region $(\mathrm{VHH})$ and are about one-tenth the size of a conventional mouse or human antibody. Affinity-matured VHHs against a target of interest can be isolated via phage display following successive antigen immunizations of llamas. This strategy has been used to isolate an influenza hemagglutinin (HA)-specific VHH that protects against infection with one influenza strain in mice ${ }^{2}$.

Laursen et al. ${ }^{1}$ immunized llamas several times with a commercial influenza vaccine containing HA proteins from three influenza $A$ and $B$ strains, along with recombinant HAs from other influenza viruses. Four VHHs were subsequently isolated: two with strong in vitro neutralizing activity against group 1 and group 2 influenza A viruses, and two with comparable activity against influenza $B$ viruses. All but one bound to epitopes on the highly conserved HA stem that are partly shared with previously described influenza broadly neutralizing antibodies.

Multimerization of VHHs specific for different epitopes is known to improve neutralization breadth against several viruses, including influenza. Similarly, Laursen et al. ${ }^{1}$ found that a multidomain antibody, MD3606, combining all four identified VHHs was superior to a previously described HA stem-binding human broadly neutralizing antibody, CR9114, in neutralizing a panel of 60 influenza $A$ and $B$ viruses in mice.

Although the binding properties of CR9114 and the individual VHHs in MD3606 appear

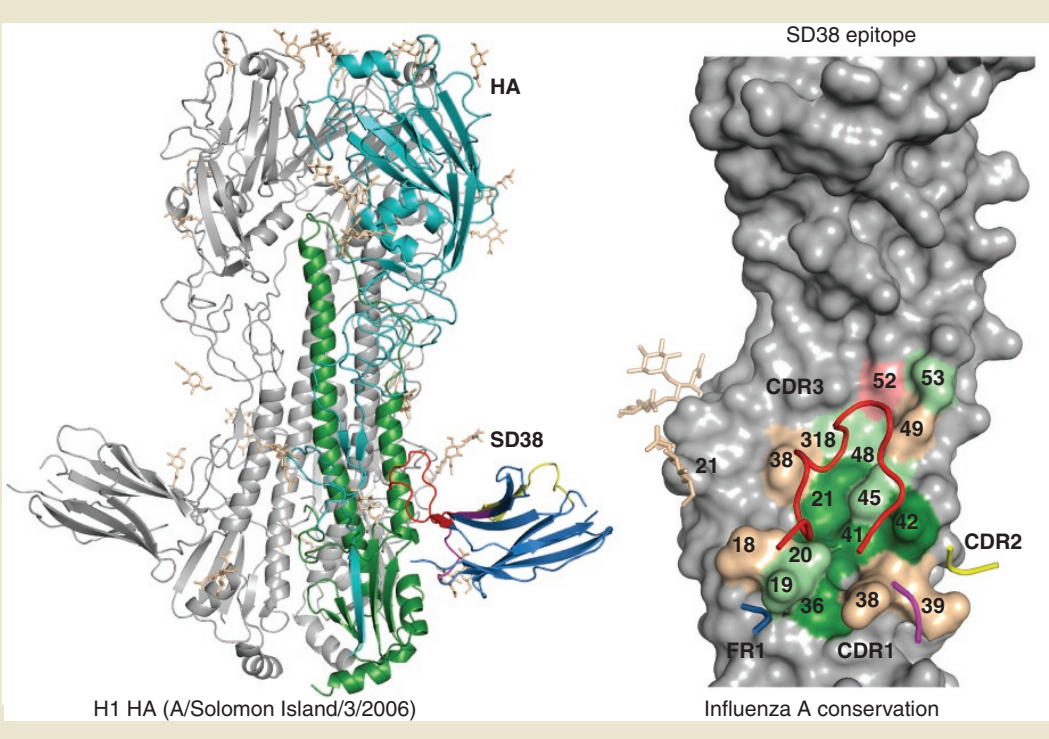

Reprinted with permission from ref. 1.

similar, according to Alejandro Balazs of the Ragon Institute, "we can't exclude the possibility that having a linear arrangement of VHHs may enhance accessibility over Fab elements of an antibody and be the underlying explanation for the high potency [of MD3606]." Structural studies of MD3606 bound to different HAs will be necessary to address this question.

Passive antibody transfer is an inefficient means of delivering antibodies as it requires large-scale protein production and multiple injections for durable protection. In contrast, AAV delivery enables stable antibody gene expression. AAV delivery of the heavy and light chains for flu-specific broadly neutralizing antibodies has been shown to completely protect against lethal doses of influenza A virus in mice ${ }^{3,4}$ and in ferrets ${ }^{4}$. However, delivering several antibody genes this way to increase neutralization breadth may be problematic, says Balazs, as this would "increase the potential of mispairing of heavy and light chains between different specificities, which could produce new antibodies with off-target effects." Additionally, the packaging capacity of AAV limits the number of genes that can be expressed on the same vector. In contrast, MD3606 contains only heavy chains and was packaged in a single AAV.

Intranasal AAV delivery as described by Laursen et al. ${ }^{1}$ may be beneficial in providing protection at the natural site of infection and may also enable long-term expression in the lungs ${ }^{5}$. In nonhuman primates, AAVmediated expression of a reporter protein in the nasal cavity waned after 3-4 months ${ }^{4}$, and it is therefore unclear whether localized AAV antibody gene delivery would provide durable protection in humans. Given the stability of serum antibody titers (at least 11 months) and the influenza protection observed in mice after intramuscular AAV gene delivery ${ }^{3}$, it will be important to evaluate the impact of the vector delivery route. Human immune tolerance of llama proteins must also be determined.

There is an urgent need for new therapeutic strategies to combat influenza, and those that can confer universal and quick protection, as MD3606 appears to, are of great interest. "It would be great to see this put into clinical trials to determine the ability of this route and vector to deliver antibody safely to patients," says Balazs. MD3606 may also serve as a template for a new type of extra-potent antibody in which VHHs specific for old and new circulating strains of a genetically diverse pathogen are delivered as a single multimerized reagent in a single injection.

\section{Saheli Sadanand, Locum Associate Editor}

1. Laursen, N.S. et al. Science 362, 598-602 (2018).

2. Ibañez, L.I. et al. J. Infect. Dis. 203, 1063-1072 (2011).

3. Balazs, A.B., Bloom, J.D., Hong, C.M., Rao, D.S. \& Baltimore, D. Nat. Biotechnol. 31, 647-652 (2013).

4. Limberis, M.P. et al. Sci. Transl. Med. 29, 187ra72 (2013).

5. Limberis, M.P. \& Wilson, J.M. Proc. Natl. Acad. Sci. USA 103, 12993-12998 (2006). 